\title{
Foundations of Social and Ethical Responsibility Among Undergraduate Engineering Students: Overview of Results
}

\section{Dr. Carla B. Zoltowski, Purdue University-Main Campus, West Lafayette (College of Engineering)}

Carla B. Zoltowski is an assistant professor of engineering practice in the Schools of Electrical and Computer Engineering and (by courtesy) Engineering Education, and Director of the Vertically Integrated Projects (VIP) Program within the College of Engineering at Purdue. She holds a B.S.E.E., M.S.E.E., and Ph.D. in Engineering Education, all from Purdue. Her research interests include the professional formation of engineers, diversity, inclusion, and equity in engineering, human-centered design, engineering ethics, and leadership.

\section{Prof. Brent K Jesiek, Purdue University at West Lafayette}

Dr. Brent K. Jesiek is an Associate Professor in the Schools of Engineering Education and Electrical and Computer Engineering at Purdue University. He also leads the Global Engineering Education Collaboratory (GEEC) research group, and is the recipient of an NSF CAREER award to study boundary-spanning roles and competencies among early career engineers. He holds a B.S. in Electrical Engineering from Michigan Tech and M.S. and Ph.D. degrees in Science and Technology Studies (STS) from Virginia Tech. Dr. Jesiek draws on expertise from engineering, computing, and the social sciences to advance understanding of geographic, disciplinary, and historical variations in engineering education and practice.

\section{Dr. Stephanie Claussen, Colorado School of Mines}

Stephanie Claussen is a Teaching Professor with a joint appointment in the Engineering, Design, and Society Division and the Electrical Engineering Department at the Colorado School of Mines. She obtained her B.S. in Electrical Engineering from the Massachusetts Institute of Technology in 2005 and her M.S. and Ph.D. from Stanford University in 2008 and 2012, respectively. Her current engineering education research interests include engineering students' understanding of ethics and social responsibility, sociotechnical education, and assessment of engineering pedagogies.

\section{Ms. Shiloh James Howland, Brigham Young University}

Shiloh James Howland is a doctoral student at Brigham Young University in Educational Inquiry, Measurement, and Evaluation. She received a master's degree in instructional psychology and technology as well as a bachelor's degree and master's degree in geology. Her current research interests are in educational measurement and assessment.

\section{Ms. Dayoung Kim, Purdue University-Main Campus, West Lafayette (College of Engineering)}

Dayoung Kim is a Ph.D. student in the School of Engineering Education at Purdue University. Her current research interest centers on engineering ethics and social responsibility, and she is specifically interested in cultural influences on engineers' moral formation. She earned her B.S. degree in Chemical Engineering at Yonsei University, South Korea in 2017.

\section{Ms. Swetha Nittala, Purdue University-Main Campus, West Lafayette (College of Engineering)}

Swetha is currently a Ph.D. student in the School of Engineering Education at Purdue. Her current work includes identifying and developing leadership and technical competencies for early-career engineers and managers. She integrates her research in Engineering Education with a prior background in Human Resource Management and Engineering to understand better ways to manage technical talent in organizations and universities. 


\section{Foundations of Social and Ethical Responsibility Among Undergraduate Engineering Students: Overview of Results}

\section{Introduction}

Because engineers have a critical role in serving the public, supporting the success of private sector enterprise, and addressing problems of global importance, many formal courses and programs have been created to promote professional responsibility and ethical integrity among engineering graduates. Other interventions (e.g., service learning programs) have also been developed to more broadly challenge engineering students to develop as engaged citizens and community members.

Yet there has been a notable lack of research on measures and understandings of social and ethical responsibility among undergraduate engineering students. Further, few studies have looked at how such indicators change over time and are impacted by specific kinds of learning experiences. To address this gap, our NSF-supported Cultivating Cultures for Ethical STEM (CCE STEM) research project has been implementing a longitudinal, mixed-methods study with collaborators and research subjects from four U.S. engineering schools. To improve transferability of results, our study includes universities of different types in different geographic locales, including public research-intensive, private research-intensive and public undergraduateserving institutions across the country.

Our study is following a cohort of students from the beginning of their enrollment in one of these engineering programs through their fourth year of study. Currently in the final year of the project, our study design included interviews and surveys in Year 1, a repeat survey administration in the students' fifth semester (Year 3), and repeat surveys and interviews during their eighth semester (Year 4). We have completed analyses of the initial and mid-point survey results and have preliminary results from the final survey administration. Our analyses of the interview data include thematic coding of how students understand ethics both in general and in engineering specifically, primary learning outcomes and influences, and evidence of moral disengagement.

In this paper, we give a summary of our previously published work and the three main analysis approaches that we are currently undertaking: investigation of the quantitative survey data, analysis of the learned outcomes that students report in their final interviews, and a phenomenographic approach to understand how the students experience ethics and social responsibility. We conclude with future work related to this project, as well as plans to continue to track our participants as they begin their careers as young professionals to understand the continued evolution of their conceptions of ethics and social responsibility.

\section{Overview of the study design}

As detailed in previous publications [1], the longitudinal study design for our project had three data collection phases covering all four years of a typical undergraduate engineering student's degree program. Our first round of baseline data collection, including survey measures and interviews, took place in Fall 2015 and Spring 2016 when our subjects were first-year students. 
Mid-point data collection, in the form of a repeat survey, was deployed in Fall 2017, or the fifth semester for the typical student participating in our study. The final phase of data collection occurred in Spring 2018, or what would typically be the eighth semester for most students in our study, and involved both repeat survey measures and follow-up interviews. An overview of the data collection process, including numbers of respondents, is shown in Figure 1.

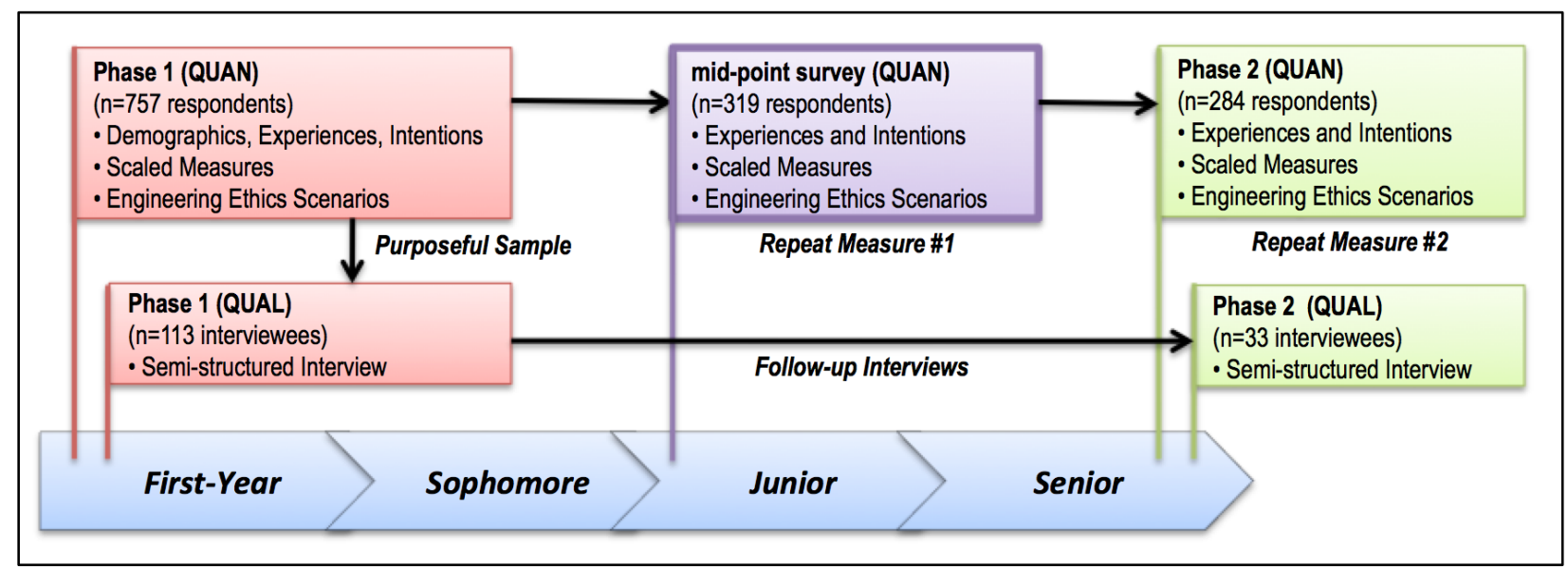

Figure 1. Overview of Data Collection Process

The survey used in this study included items and measures related to engineering ethics knowledge, justice beliefs, political and social involvement, macro-ethical considerations, moral attentiveness, moral disengagement, and ethical climate, along with extensive demographic questions. More information about the measures can be found in other papers by our team cited below. A semi-structured interview protocol was also used to collect qualitative data from select student participants and included questions related to: 1) general definitions of ethics and engineering ethics, including macro-ethics, 2) experiences (past, present, and future) that may shape students' ethical perspectives and sense of social responsibility, 3) ethical climate, 4) ethical scenarios, and 5) select survey items identified as promising for further probing.

The four participating universities involved in this study represent different geographic locales and university types, including a public research-intensive and project-based university in the Southwest (Arizona State University); a private, religious, and research-intensive university located in the Mountain West (Brigham Young University); a public, primarily undergraduateserving university located in the Mountain West (Colorado School of Mines); and a public research-intensive university located in the Midwest (Purdue University). All data was carried out at each school under appropriate human subject research protections and IRB approvals.

\section{Summary of results reported in previous conference papers}

Here we offer a brief guide to previously published results for our project. To begin, overall findings related to our survey data has appeared in two papers. The first summarized results for all respondents and all survey measures [2], reporting significant variability in results for multiple measures among domestic versus international students, and by perceived levels of importance of religion in students' lives. A second paper reported on trends from the baseline to mid-point survey, with particular emphasis on examining variations by student-level variables 
such as university affiliation, demographics, importance of religion, and involvement in various kinds of learning experiences [3]. Significant findings include evidence of general gains on an ethics knowledge measure, decreased perceptions of ethical climate across schools, decreases in a Justice for Self measure, and higher levels of both moral attentiveness and political and social involvement among students who were in more socially-oriented engineering fields.

Findings from the qualitative (interview) data have been reported in a parallel set of papers. The first introduces the codebook used to analyze the first set of interview data [4]. This paper also reports preliminary findings for a subset of that data ( $n=29$ interviews) with emphasis on three themes: how students understand and define moral/ethical character in general, how they understand engineering ethics in particular, and evidence of specific learned outcomes related to ethics/morality. This paper also explores some common threads across the three areas, including student awareness about the potential consequences or impacts of ethical decisions, the role of integrity and honesty in ethics and morality, and the importance of other-oriented traits like empathy. A second paper, based on 66 first phase interviews, further examined specific learned outcomes associated with various influences and learning experiences (e.g., academic curricular, extracurricular activities, family, etc.) [5]. The findings underscore the extent to which students learn lessons related to ethics from nearly every facet of their lives and through a wide range of modalities. Finally, a third paper found considerable evidence of moral emotion (both positive and negative) and moral intuition based on an in-depth analysis of 11 first-phase interviews [6].

Two other papers involved use of mixed-method approaches to more deeply investigate specific constructs. The first of these was focused on moral disengagement based on survey and interview data from the study's first phase [7]. Notable findings include similar or lower moral disengagement scores among our student respondents as compared to other studies, and evidence of how students applied different ethical frames when evaluating moral situations and/or making moral decisions. Further, we observed that seven of the eight types of moral disengagement measured by the survey instrument were also evident in our interview data, and we additionally proposed a novel mechanism called "unreflective disengagement." Yet another paper examined changing perceptions of ethical climate based on surveys and select interviews from the initial and final phases of data collection [8]. In addition to reporting a decline in ethical climate perceptions among survey respondents, this paper used interview data to explore how this trend may be linked to the climate and culture of engineering departments and degree programs.

\section{Quantitative, longitudinal analysis of measures of ethics and social responsibility}

The major quantitative aspect of this project is a survey that was administered to eligible engineering students at three time points. During their senior year (Year 4), we contacted the students who had taken our initial survey in 2015 (Year 1) and again asked them to respond to our survey for a third time. These final responses were linked to their initial responses (Year 1) and to their mid-point responses (Year 3). Of the original 757 students who responded to the initial survey, 284 students responded to the final survey and of those, 226 responded to all three surveys.

This survey was composed of eight scales (89 items) and also asked them to indicate if they had participated in a variety of experiences during college. These scales included measures of 
situational judgment, their perceptions of their university's ethical climate, justice beliefs, the Political and Social Involvement Scale (PSIS), a series of items related to macro-ethical considerations, moral attentiveness, and moral disengagement. Students also rated the importance of technical, ethical, economic, health and safety, environment, social and manufacturability considerations. We also asked the students, on the mid-point and final surveys, to indicate specific experiences they had participated in at any point in their undergraduate, including: volunteering regularly, completing a mission or volunteering trip, working or interning for a non-profit, government, or engineering-related organization, taking a servicelearning course, travel to a developing country, involvement in student government, receiving formal religious or ethical instruction, participating in an honors program or service-focused extracurricular organization (e.g., Engineers Without Borders), joining an engineering professional society, completing an undergraduate research experience, participating in a fraternity or sorority, attending a study abroad program, and participating in a Grand Challenges Scholars Program. The 226 students that completed the survey three times make up the sample that was analyzed to look for changes over time on their scores on these eight scales, as well as to look for differences by gender, university, or participation in the experiences listed above. Detailed findings from analysis of this data will be reported in a paper now under development.

\section{Qualitative analysis of engineering students' learning of ethics}

One of the main objectives of this study is to identify specific types of experiences or interventions that have positive impacts on engineering students' understanding of ethical and social responsibility. To identify such experiences, we focused on Year 4 (phase 2) interview data, because senior engineering students are in the most developed stage in our longitudinal investigation timeframe. If we understand what experiences - either inside or outside of college - students in Year 4 perceive as influential factors in their learning of ethics, as well as what the students learned as a result, it could provide insights on how to design educational interventions for engineering students' learning of ethics.

We analyzed all 33 interviews of phase 2 and identified (coded) students' self-identified contributors to their ethics learning as "Influences". This coding category included several subcategories (codes): Academics, Extracurricular, Family, International, Professional/work, Religion, Service/Volunteer, and Social/friends. In addition, we identified (coded) instances of all explicit or implicit statements about insights, learning, or realizations related to ethics, morality, values, etc. These statements often included phrases such as "that made me see", "I realized that", or "that taught me that." We are currently analyzing these coded statements to understand relationships between the content (what students learned) and the mechanism (how students learned) of their learning. Again, these findings will be reported in a manuscript that is currently under development.

\section{Phenomenography: Understanding students' perceptions of ethics and social responsibility}

During our preliminary analysis of the phase 1 interview data, we noted that students had varied understandings of ethics and social responsibility. In addition, these variations in experiences and perceptions could not be mapped onto a prior existing framework. Hence, in order to better understand and capture all the different ways in which students' experience ethics and social 
responsibility in a theoretically grounded approach, we decided to incorporate a phenomenographic approach to our phase 2 data collection and analysis efforts.

Phenomenography is a qualitative and empirical research method that emerged from educational research $[9,10]$. Engineering education researchers have used phenomenography to explore complex engineering activities (phenomenon) such as human-centered design [11], crossdisciplinary teamwork [12], and innovation [13]. Consequently, in phase 2, the interview protocol was modified to include questions to explore the qualitatively different ways in which the students experienced engineering ethics. The initial prompt was: "Can you describe an experience you have had with an ethical situation as an individual, student, and/or an aspiring professional?", which was followed by a series of questions to elicit more discussion of the participant's experience of the ethical situation.

The phenomenographic analysis process, which is iterative and comparative, began by one author reading and re-reading the data pertaining to the phenomenographic questions from all the 33 interviews of phase $2[14,15,16]$. Considering the data from each participant holistically and in the context of the other participants, the transcripts were sorted into initial categories. Descriptions of these categories that identified similarities within each category, and differences between the categories were presented to other members of the research group. Multiple iterations of this process will be completed until the categories and the structural relationship between them converges. This analysis is ongoing with the aim of reporting our findings in a future journal paper.

\section{Future work}

In addition to what is described about, our future work plans also include efforts to synthesize and connect findings from across the different stages and facets of the study, including both the quantitative and qualitative data sets. This synthesis will prioritize the findings from the three studies discussed in this paper as they represent culminating perspectives of the students on their undergraduate experiences. In addition, we plan to make information about our longitudinal dataset available for potential use by other researchers.

We also plan to build upon the data gathered from our current longitudinal study by following our current cohort into the first years of their professional careers. In addition, we plan to expand the pool of survey respondents and interviewees to other young engineers at approximately the same point in their careers. Our planned study will extend understanding of how the experiences students participate in during their time as undergraduates impacts their beliefs around ethics and social responsibility as young professionals, while also seeing how their early career experiences (e.g., in graduate school, corporate work settings, etc.) change their perceptions and beliefs. Furthermore, this continuation of our study will allow us to explore how institutional and cultural factors (such as engineering discipline, industry, and company/organization) influence perceptions of ethics and social responsibility among early career engineering professionals.

\section{Acknowledgments}

This material is based upon work supported by the National Science Foundation under Grant Nos. 1449370, 1449470, 1449479, and 1449490. Any opinions, findings, and conclusions or 
recommendations expressed in this material are those of the author(s) and do not necessarily reflect the views of the National Science Foundation.

\section{References}

[1] C. B. Zoltowski, B. K. Jesiek, S. A. Claussen, and D. H. Torres, "Foundations of Social and Ethical Responsibility Among Undergraduate Engineering Students: Project Overview," in Proceedings of the 2016 ASEE Annual Conference and Exposition, June 26-29, 2016, New Orleans, LA, USA. [Online]. Available: https://peer.asee.org/foundations-of-social-and-ethical-responsibility-amongundergraduate-engineering-students-project-overview

[2] D. S. Fuentes, G. M. Warnick, B. K. Jesiek, and R. Davies, “A Longitudinal Study of Social and Ethical Responsibility Among Undergraduate Engineering Students: Preliminary Survey Results," in Proceedings of the ASEE Annual Conference and Exposition, June 26-29, 2016, New Orleans, LA, USA. [Online]. Available: https://peer.asee.org/a-longitudinal-study-of-social-and-ethical-responsibility-amongundergraduate-engineering-students-preliminary-results

[3] S. M. J. Howland, G. M. Warnick, C. B. Zoltowski, B. K. Jesiek, and R. Davies, “A Longitudinal Study of Social and Ethical Responsibility Among Undergraduate Engineering Students: Comparing Baseline and Midpoint Survey Results" in Proceedings of the ASEE Annual Conference and Exposition, June 24-27, 2018, Salt Lake City, UT, USA. [Online]. Available: https://peer.asee.org/a-longitudinal-study-of-social-andethical-responsibility-among-undergraduate-engineering-students-comparing-baselineand-midpoint-survey-results

[4] B. K. Jesiek, C. B. Zoltowski, D. S. Fuentes, S. Claussen, and G. M. Warnick, "Investigating Engineering Students' Understandings of Social and Ethical Responsibility: Coding Framework and Initial Findings," in Proceedings of the 2017 ASEE Annual Conference and Exposition, June 25-28, 2017, Columbus, OH, USA. [Online]. Available: https://www.asee.org/public/conferences/78/papers/19677/download

[5] S. Nittala, T. Zephirin, S. M. J. Howland, D. Kim, A. Katz, and B. K. Jesiek, "Investigating Influences on First-year Engineering Students' Views of Ethics and Social Responsibility" in Proceedings of the ASEE Annual Conference and Exposition, June 24-27, 2018, Salt Lake City, UT, USA. [Online]. Available: https://peer.asee.org/investigating-influences-on-first-year-engineering-students-viewsof-ethics-and-social-responsibility

[6] D. Kim and B. K. Jesiek, "Work-in-Progress: Emotion and Intuition in Engineering Students' Ethical Decision Making and Implications for Engineering Ethics Education" in Proceedings of the ASEE Annual Conference and Exposition, June 15-19, 2019, Tampa, FL, USA. [Online]. Available: https://www.asee.org/file_server/papers/attachment/file/0010/7369/_Final_2019_ASEE Emotion and Intuition 
[7] D. Kim, P. W. Odom, C. B. Zoltowski, and B. K. Jesiek, "Investigating Moral Disengagement Among First-Year Engineering Students," in 2018 IEEE Frontiers in Education Conference (FIE), Oct. 3-6, 2018, San Jose, CA, USA.

[8] S. Nittala, S. M. J. Howland, and B. K. Jesiek, "Changes in Perceptions of Ethical Climate Among Undergraduate Engineering Students," in 47th SEFI Annual Conference, Sept. 16-19, 2019, Budapest, Hungary.

[9] S. Booth, "On Phenomenography, Learning and Teaching," Higher Education Research \& Development, vol. 16, no. 2, pp. 135-158, 1997.

[10] F. Marton, "Phenomenography - A research to investigating different understandings of reality," Journal of Thought, vol. 21, no. 3, pp. 28-49, 1986.

[11] C. B. Zoltowski, W. C. Oakes, and M. E. Cardella, "Students' ways of experiencing human-centered design," Journal of Engineering Education, vol. 101, no. 1, pp. 28-59, Jan. 2013.

[12] E. Dringenberg, J. A. Mendoza-Garcia, M. Tafur-Arciniegas, N. D Fila, and M.-C. Hsu, "Using phenomenography: Reflections on key considerations for making methodological decisions" in Proceedings of the ASEE Annual Conference and Exposition, June 14-17, 2015, Seattle, WA, USA. [Online]. Available: https://peer.asee.org/usingphenomenography-reflections-on-key-considerations-for-making-methodologicaldecisions

[13] N. D. Fila and Ş Purzer, "Work in progress: A preliminary investigation of the ways engineering students experience innovation," in Proceedings of the ASEE Annual Conference and Exposition, June 26-29, 2016, New Orleans, LA, USA. [Online]. Available: https://peer.asee.org/work-in-progress-a-preliminary-investigation-of-theways-engineering-students-experience-innovation

[14] G. S. Ákerlind, "Variation and commonality in phenomenographic research methods." Higher Education Research and Development, vol. 24, no. 4, pp. 321-334, 2005.

[15] J. A. Bowden and P. Green, Doing Developmental Phenomenography. Melbourne, Australia: RMIT University Press, 2005.

[16] F. Marton and S. Booth, Learning and Awareness. Mahwah, NJ, USA: Lawrence Erlbaum Associates, 1997. 